\title{
Klebsiella pneumoniae: Prevalence of ESBL Producing Clinical Isolates and their Antimicrobial Susceptibility Pattern in a Hospital Setting
}

\author{
B. Sandya Gnana Sailaja and A. Heraman Singh* \\ Department of Microbiology, GSL Medical College, Rajahmundry - 533296, India \\ *Corresponding author
}

\section{A B S T R A C T}

The incidence of infections caused by multidrug resistant ESBL producing K.pneumoniae is on rise. The treatment of these infections have become

Keywords

ESBL producing

K.pneumoniae, cephalosporins, piperacillin/tazobact am, carbapenems

Article Info

Accepted:

28 June 2019

Available Online:

10 July 2019 increasingly difficult and life threatening. Knowledge on prevalence of these infections and corresponding antimicrobial susceptibility patterns is essential regionally. Routine standard operative procedures were followed in the laboratory in processing the clinical specimens for culture and sensitivity. ESBL producing K.pneumoniae were identified by phenotypic combination disc test. Majority of K.pneumoniae were isolated from respiratory specimens and pus, followed by urine and blood respectively. $42(40.38 \%)$ isolates were confirmed as ESBL producing K.pneumoniae. The resistance demonstrated to $3^{\text {rd }}$ generation cephalosporins was significant and to non $\beta$ lactam antibiotics was moderate. Resistance exhibited to piperacillin/tazobactam was low and nil to carbapenems and tigecycline. The infections caused by multidrug resistant ESBL producing K.pneumoniae are significant. Due to their multidrug resistant nature the treatment options available are limited. Therefore, active surveillance and appropriate infection control measures are necessary to control the spread of these pathogens.

\section{Introduction}

Klebsiella pneumoniae causes a variety of infections, ranging from pneumonia, urinary tract infection, bacteremia, meningitis, sepsis and abscesses. In the past, K. pneumoniae has caused serious infections mainly in immunodeficient individuals and in those with comorbid diseases. But, in the recent times due to the rise of hypervirulent strains, the frequency and severity of infections has increased in healthy and immunocompetent individuals also. ${ }^{1}$ Furthermore, in recent decades, the incidence of infections caused by multidrug resistant extended spectrum $\beta$ lactamase (ESBL) producing K.pneumoniae is on rise. Due to this, the treatment of these infections have become increasingly difficult and life threatening. ${ }^{2,3}$

The resistance to multiple antibiotics seen in K.pneumoniae is due to the ability to produce enzymes like ESBLs and carbapenemase. ESBL producing strains exhibit resistance to 
cephalosporins and monobactams. Carbapenemase producing strains show resistance to nearly all the available beta lactams, including the carbapenems. ${ }^{4}$ Due to the lack of effective treatments the infections caused by these multi drug resistant strains along with the possibility of subsequent bacteremia and sepsis are associated with higher rates of morbidity and mortality. ${ }^{5}$

The rise in incidence of hypervirulent strains, spread of resistance to multiple antibiotics, the morbidity and mortality associated with infections by these strains and the cost of management warrant efforts to better understand the epidemiology of K.pneumoniae. Knowledge on prevalence of K.pneumoniae infections and corresponding antimicrobial susceptibility patterns is essential particularly in our region where the information available is insufficient to guide in the management of these infections.

\section{Materials and Methods}

This observational cross section study was carried out at GSL general hospital, Rajamahendravaram, Andhra Pradesh, India between September 2018 and December 2018. Routine standard operative procedures were followed in the laboratory in processing the clinical specimens for culture and sensitivity that were obtained from patients admitted in the hospital. ${ }^{6,7,8,9}$

Antimicrobial susceptibility test was done on Mueller-Hinton agar medium by the conventional modified Kirby-Bauer disc diffusion method. Disks Commercially available from HIMEDIA containing a known concentration of antimicrobial agent were used in the test. CLSI recommendations were followed.

Detection of ESBL producing K.pneumoniae was done by phenotypic combination disc test. The isolates that have demonstrated zones of inhibition to cefotaxime $\leq 27 \mathrm{~mm}$ and to ceftazidime $\leq 22 \mathrm{~mm}$ in the initial screen test were subjected to combination disc test using both cefotaxime and ceftazidime, alone and in combination with clavulanic acid. Then the zone of inhibition to cephalosporin disc with clavulanic acid was compared with the zone of inhibition to cephalosporin alone (Figure.1). The isolate was considered as ESBL producer when the inhibition zone diameter was $\geq 5 \mathrm{~mm}$ larger with clavulanic acid than without. Quality control: K.pneumoniae ATCC 700603. ${ }^{10}$

\section{Results and Discussion}

A total number of 926 clinical specimens were processed for culture and sensitivity during the study period. Bacterial culture was positive in 688 clinical specimens and out of them $104(15.11 \%)$ isolates were identified as K.pneumoniae (Figure.2). Majority of K.pneumoniae were isolated from respiratory specimens (42) and pus (37), followed by urine (21) and blood (4) respectively (Table 1). Among these 104 clinical isolates, 42 (40.38\%) were confirmed as ESBL producing strains of K.pneumoniae (Figure. 3).

All the strains of ESBL producing K.pneumoniae have demonstrated resistance to ampicillin, amoxicillin/clavulanate, cefazolin, ceftriaxone, ceftazidime and aztreonam. Resistance to Piperacillin/ tazobactam exhibited was $12 \%$ and to carbapenems was nil. Resistance noted to non $\beta$ lactam antibiotics like aminoglycosides was $40.5 \%$, quinolones was $50 \%$, cotrimoxazole was $42.8 \%$ and tigecycline was nil (Table.2).

K.pneumoniae is a common cause of bacterial infections in hospitalized individuals. It is seen in water and soil and it readily colonizes mucosal surfaces of human gastrointestinal tract, conjunctiva, respiratory tract, and the 
genitourinary tract. ${ }^{11}$ From these sites, it can gain access to other tissues and cause severe infections in humans.

Epidemiological studies indicate that the inappropriate use of $3^{\text {rd }}$ generation cephalosporins has resulted in the emergence of multidrug resistant ESBL producing K.pneumoniae. ${ }^{12}$ In the present study, the prevalence of these pathogens was noted as $40.38 \%$. The prevalence varies between hospitals as it depends on various factors like infection control measures, antibiotic policy and carriage rate of these pathogens among the patients and hospital personal.
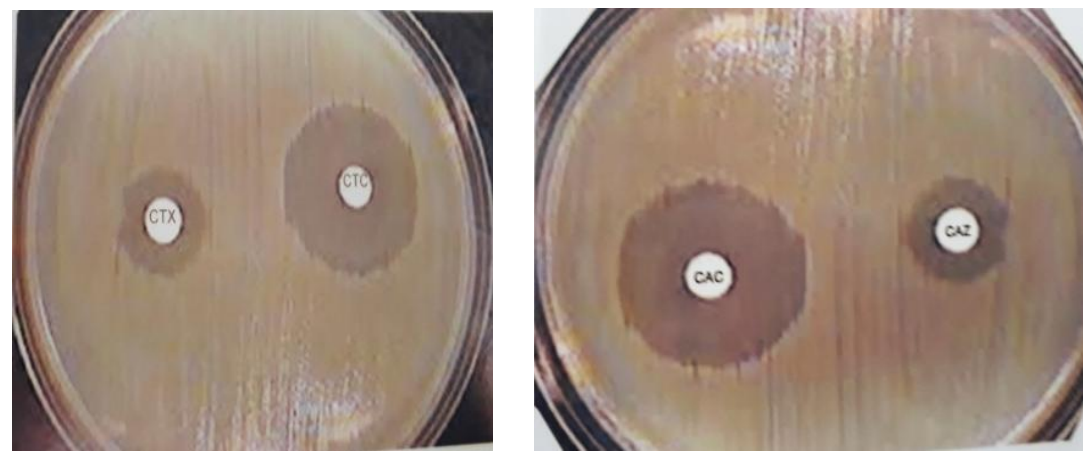

Figure.1 ESBL detection by phenotypic combination disc test

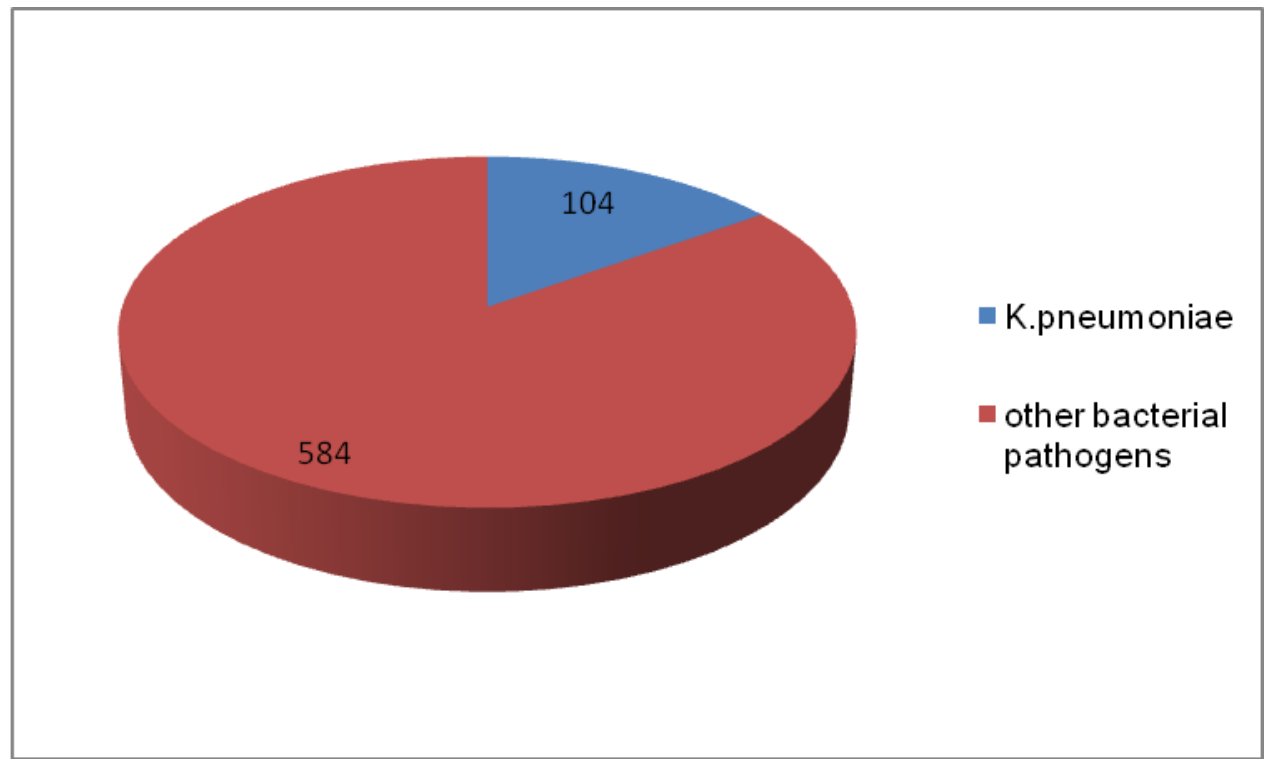

Figure.2 Frequency of Klebsiella pneumoniae among the study group $(\mathrm{n}=688)$ 


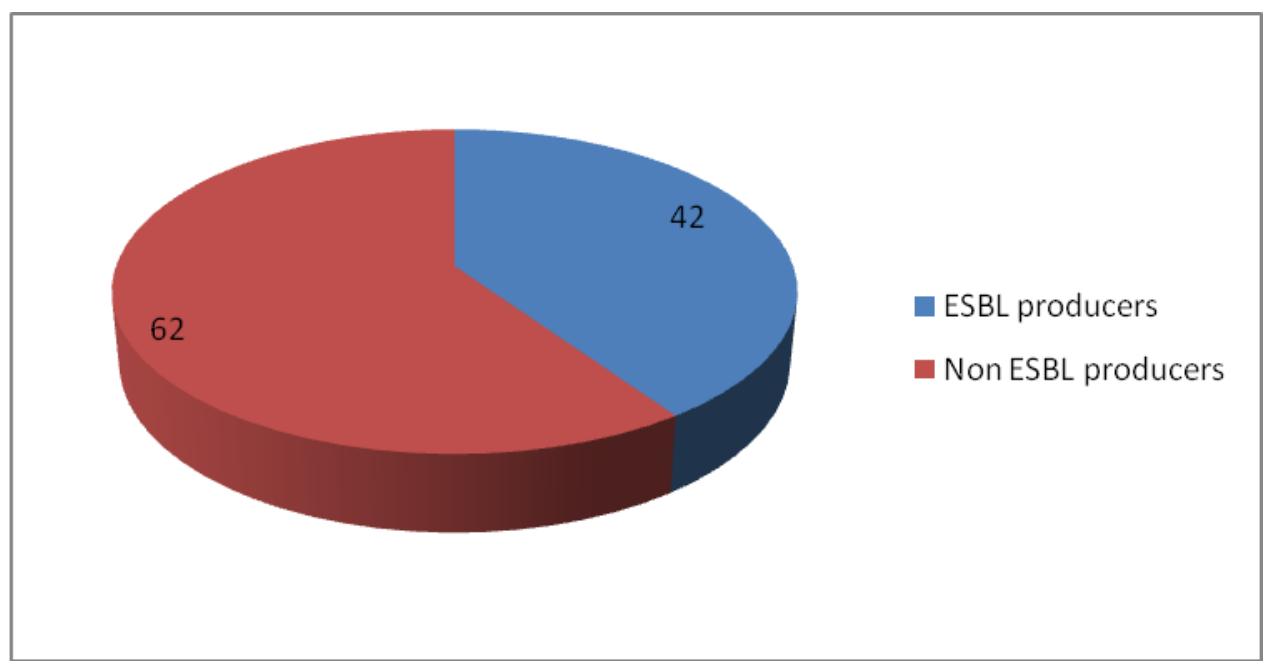

Figure.3 Frequency of ESBL producing Klebsiella pneumoniae among the study group $(\mathrm{n}=104)$

Table.1 Clinical site wise distribution of Klebsiella pneumoniae among the study group (n=104)

\begin{tabular}{|c|l|c|c|}
\hline S.No & Clinical site involved & No.of isolates & $\%$ \\
\hline $\mathbf{1}$ & Respiratory specimen & 42 & 40.38 \\
\hline $\mathbf{2}$ & Pus & 37 & 35.57 \\
\hline $\mathbf{3}$ & Urine & 21 & 20.19 \\
\hline $\mathbf{4}$ & Blood & 4 & 3.84 \\
\hline
\end{tabular}

Table.2 Antibiotic susceptibility pattern among ESBL producing Klebsiella pneumoniae $(\mathrm{n}=42)$

\begin{tabular}{|c|c|c|c|}
\hline S. No & Drug name (Strength $\mu \mathrm{g}$ ) & $\begin{array}{l}\text { Sensitivity } \\
(\%)\end{array}$ & $\begin{array}{l}\text { Resistance } \\
\text { (\%) }\end{array}$ \\
\hline 1 & Cotrimoxazole (25) & 57.2 & 42.8 \\
\hline 2 & Gentamicin (10) & 59.5 & 40.5 \\
\hline 3 & Amikacin (30) & 59.5 & 40.5 \\
\hline 4 & Tobramycin (10) & 59.5 & 40.5 \\
\hline 5 & Tigecycline (15) & 100 & 0 \\
\hline 6 & Aztreonam (30) & 0 & 100 \\
\hline 7 & $\begin{array}{l}\text { Ampicillin (10) and Amoxicillin- } \\
\text { clavulanic acid (20/10) }\end{array}$ & 0 & 100 \\
\hline 8 & $\begin{array}{l}\text { Cefazolin }(30) \text {, ceftriaxone }(30) \text {, } \\
\text { ceftazidime }(30)\end{array}$ & 0 & 100 \\
\hline 9 & Ciprofloxacin (5) & 50 & 50 \\
\hline 10 & Levofloxacin (5) & 50 & 50 \\
\hline 11 & Piperacillin-Tazobactam (100/10) & 88 & 12 \\
\hline 12 & Imipenem (10) & 100 & 0 \\
\hline 13 & Meropenem (10) & 100 & 0 \\
\hline
\end{tabular}


ESBL producing K.pneumoniae pose challenging situations during the treatment of infections caused by these multidrug resistant strains. Due to the rise of these strains the effectiveness of broad spectrum cephalosporins is restricted.

High level resistance demonstrated to cefotaxime $(70 \%)$ and ceftazidime $(75 \%)$ in this study therefore indicate that these broad spectrum antibiotics are empirically not recommended in the management of serious infections caused by these pathogens. ${ }^{17}$

Piperacillin and tazobactam combination is a broad spectrum antibiotic against a variety of bacterial pathogens including ESBL producing organisms. Low level resistance (12\%) exhibited to this combination may be examined as a choice of antibiotic in the empirical treatment of serious infections caused by these pathogens. However, it should be used with a caution as susceptibility in vitro may not necessarily estimate the effectiveness in vivo. ${ }^{18}$

All the K.pneumoniae isolates were sensitive to imipenem and meropenem. Carbapenems are valuable against ESBL producing organisms as they are resistant to ESBL enzymes and also due to their small size they can easily enter through the porins into the periplasmic space of Gram negative bacilli. Thus, carbapenems are usually evaluated empirically in the treatment of serious infections caused by these pathogens. However, liberal and widespread use of these drugs might facilitate the emergence of carbapenem resistance. ${ }^{19}$

Resistance level shown to non $\beta$ lactam antibiotics such as aminoglycosides, quinolones and cotrimoxazole was moderate ranging from $40.50 \%$ to $50 \%$. This could be due to pathogens possessing the plasmids with resistance genes that encode ESBL genes also carry resistance genes to other groups of antibiotics. Thus, these pathogens are multidrug resistant and therefore reduce the treatment options for infections caused by these pathogens to carbapenems and newer drugs like tigecycline. ${ }^{18,19}$

In conclusion, the infections caused by ESBL producing K.pneumoniae in our hospital setting is significant. Due to their multidrug resistant nature the treatment options available are restricted. Therefore, active surveillance for these organisms should be conducted regularly which helps in designing more effective antibiotic policies. Appropriate infection control measures like effective hand hygiene and contact precautions should be strictly implemented to control the rise and spread of these organisms.

\section{References}

1. Paczosa MK and Mecsas J, 2016. Klebsiella pneumoniae: going on the offense with a strong defense. Microbiol Mol Biol Rev. 80,629 - 661. doi:10.1128/MMBR.00078-15.

2. Boucher HW, Talbot GH, Bradley JS, Edwards JE, Gilbert D, Rice LB, Scheld M, Spellberg B Bartlett J, 2009. Badbugs, nodrugs: noESKAPE! An update from the Infectious Diseases Society of America. Clin Infect Dis. 48: 1 - 12. http://dx.doi.org/10.1086/595011.

3. Kuehn BM, 2013. Nightmare bacteria on the rise in US hospitals, long-term care facilities. JAMA. 309, 1573 - 1574. http://dx.doi.org/10.1001/jama.2013.292 2.

4. CDC, 2015. CDC works $24 / 7$ to protect US from health, safety and security threats. CDC, Atlanta, GA.

5. CDC, 2013. Vital signs: carbapenemresistant Enterobacteriaceae. MMWR Morb Mortal Wkly Rep 62:165-170.

6. Monica Cheesbrough, 2002. District laboratory practice in tropical countries. Part 2. Cambridge University Press. 
7. Elmer W. Koneman, Stephen D. Allen, William M. Janda, Paul C, 1997. Schreckenberger, Washington C. Winn, Jr. Color atlas and textbook of diagnostic microbiology. Fifth edition, Lippincott.

8. J.G. Colle, A.G. Fraser, B.P. Marmion, A.Simmons, 2008. Mackie \& McCartney Practical medical microbiology. Fourteenth edition, Elsevier.

9. Patricia M. Tille. Bailey \& Scott's Diagnostic Microbiology, 2014. Thirteenth edition, Elsevier.

10. CLSI, 2016. Performance Standards for Antimicrobial Disk Susceptibility Tests: Approved Standard - Twelfth Edition. CLSI document Mo2 - A12. Wayne, PA: Clinical and Laboratory Standards Institute.

11. Podschun R and Ullmann U, 1998. Klebsiella spp. As nosocomial pathogens: epidemiology, taxonomy, typing methods, and pathogenicity factors. Clin Microbiol Rev. 11 (4), $589-$ 603.

12. Sirot D 1995. Extended spectrum plasmid mediated $\beta$-lactamases. J Antimicrob Chemother. 36 (A), 19-34.

13. Sarojamma V and Ramakrishna V, 2011. Prevalence of ESBL producing Klebsiella pneumoniae isolates in tertiary care hospital. ISRN Microbiology. 2011, 318348. doi: 10.5402/2011/318348

14. Goyal A, Prasad KN, Prasad A, Gupta S, Ghoshal U, Ayyagari A, 2009. Extended spectrum $\beta$ lacatamases in Escherichia coli \& Klebsiella pneumoniae \& associated risk factors. Indian J Med Res. 129, 695 - 700.
15. Venezia RA, Scarano FJ, Preston KE, Steele LM, Root TP, Limberger R, Archinal W, Kacica MA.1995. Molecular epidemiology of an SHV-5 extended spectrum $\beta$ lacatamase in enterobacteriaceae isolated from infants in a neonatal intensive care unit. Clin Infect Dis. 21 (4), 915 - 923.

16. Lautenbach E, Patel JB, Bilker WB, Edelstein PH, Fishman NO, 2001. Extended spectrum $\beta$ lacatamase producing Escherichia coli \& Klebsiella pneumoniae: risk factors for infection and impact of resistance on outcomes. Clin Infect Dis. 32, 1162 - 1171.

17. Paterson DL, Ko WC, Von Gottberg A, Casellas JM, Mulazimoglu L, Klugman KP, Bonomo RA, Rice LB, McCormack JG, Yu VL, 2001. Outcome of cephalosporin treatment for serious infections due to apparently susceptible organisms producing extended spectrum $\beta$ lacatamases: implications for the clinical microbiology laboratory. J Clin Microbiol. 39, 2206 2212.

18. Gupta A, Ampofo K, Rubenstein D, Saiman L, 2003. Extended spectrum $\beta$ lactamase producing Klebsiella pneumoniae infections: a review of literature. Journal of Perinatology. 23, $439-443$.

19. Swathi CH, Rosy Chikkala, K.S. Ratnakar, V. Sritharan, 2016. A structural, epidemiological \& genetic overview of Klebsiella pneumoniae carbapenemases (KPCs). Indian J Med Res. 144, $21-31$.

\section{How to cite this article:}

Sandya Gnana Sailaja, B and Heraman Singh, A. 2019. Klebsiella pneumoniae: Prevalence of ESBL Producing Clinical Isolates and Their Antimicrobial Susceptibility Pattern in a Hospital Setting. Int.J.Curr.Microbiol.App.Sci. 8(07): 2794-2799.

doi: https://doi.org/10.20546/ijcmas.2019.807.349 